\title{
SWOT analysis of wastewater heat recovery systems application
}

\author{
Sabina Kordana ${ }^{1, *}$ \\ ${ }^{1}$ Rzeszow University of Technology, Department of Infrastructure and Sustainable Development, \\ al. Powstańców Warszawy 6, 35-959 Rzeszów, Poland
}

\begin{abstract}
Limited availability of fossil fuels and various issues connected with environment conservation lead to a growing interest in unconventional sources of energy. The latter include wastewater carried via sewage systems. Yet, the possibility to extract thermal energy from this source depends on a number of factors, location of heat recovery system being one of them. Viability of utilizing raw wastewater and effluent discharged to a receiving body after treatment process, as potential sources of energy, was assessed using SWOT/TOWS analysis. Strategies for developing systems of heat recovery from both types of wastewater have been defined, and recommendations related to their use in Poland have been formulated.
\end{abstract}

\section{Introduction}

In recent years there has been visible increase in the density of sewer networks [1]. In Poland this trend can be observed in both urban and rural areas, and one of its consequences is an increase in the volume of household and industrial wastewater carried by intercepting sewers. Such wastewater is recognized as one of the main point sources of freshwater pollution $[2,3]$, and its treatment to a required level is frequently a complicated and costly process [4]. Yet, wastewater produced by households and commercial establishments should not be seen exclusively as a problem, because it carries significant amounts of waste thermal energy which can effectively be recuperated and utilised in warming up water and heating indoor spaces. In the summer it can be used as a cold source for air-conditioning.

The need to utilize unconventional sources of energy, such as e.g. wastewater carried via intercepting sewers, is a consequence of excessive exploitation of fossil fuels. Of no small importance are also various issues connected with environment conservation as well as problems linked with climate change, whose results affect other domains of life $[5,6]$. In Poland the proportional share of unconventional energy in the total energy consumption is growing, yet coal continues to be the main source of energy for district heating systems [7]. This situation is mainly caused by a lack of systematic knowledge related to the possible utilization of waste energy contained for instance in wastewater, as a result of which it is unproductively released into the environment [8].

*Corresponding author: sk@prz.edu.pl 
In response to the above problem, the paper characterises raw and treated wastewater, from the viewpoint of their possible utilization as a source of thermal energy. The factors defined for this purpose include strengths and weaknesses of such heat recovery systems as well as potential opportunities and threats which may occur throughout the lifespan of such installations. SWOT analysis was applied as a tool, and its assumptions are described in the following section.

\section{Research Methodology}

The findings described in the paper have been acquired using SWOT analysis. This is one of the basic methods applied in strategic planning, and it can be used to assess any situation where strategic operations are required. It is assumed that this research method enables identification of factors characterising an entity or enterprise under consideration, in the context of a specified purpose, as well as classification of such factors into four groups. Two of these comprise positive, and the other two negative elements, as it was shown in Fig. 1 [9].

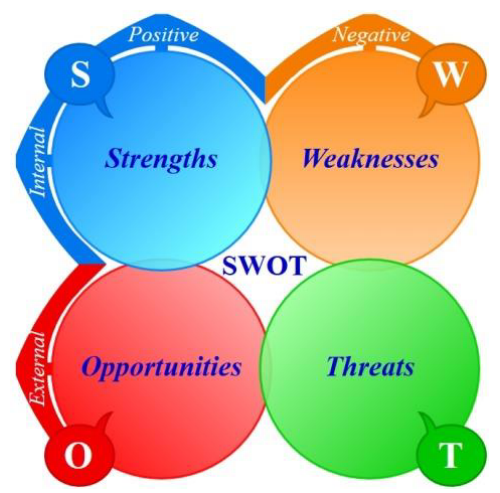

Fig. 1. SWOT analysis diagram.

The defined factors are assigned with weighting coefficients whose total within a given group should equal 1.0; additionally, interactions occurring between internal and external factors are determined. SWOT analysis is designed to examine relationships "from the inside towards the outside". Its purpose is to show, whether the identified strengths will make it possible to take advantage of the potential opportunities and to overcome the threats, and secondly to determine whether the specific weaknesses will magnify the threats and hinder the use of the opportunities [10].

SWOT analysis is supplemented with TOWS analysis, which is designed to identify interactions "from the outside towards the inside". As a result of its application it is possible to show whether the occurring opportunities will enhance the strengths and reduce the weaknesses, and secondly whether the possible threats will magnify the weaknesses and decrease impact of the strengths.

Based on the comprehensive findings of SWOT/TOWS analysis it is possible to determine development strategy for a given entity or enterprise and to define further recommendations [10].

\section{Wastewater heat recovery}

The idea of utilizing heat carried by wastewater was born a few decades ago, yet due to significant investment costs and poor efficiency of equipment in those times, the concept 
failed to gain recognition among investors and designers of energy systems. Today it is being investigated again, and the fact mainly results from the limited availability of fossil fuels. Other important reasons include the rapid developments in waste heat recovery technologies, as a result of which heat stored in wastewater may be recovered both in buildings [11] and during its transport via sewer systems [12].

In the first case, greywater from shower is usually the source of heat and the energy recovered from waste by heat exchanger is transferred to water carried by incoming water pipes [13]. On the other hand when energy is carried by wastewater transported via intercepting sewers, heat pumps or large exchangers installed inside or outside the sewer may be used; depending on the conditions in which the process is conducted the latter may function as an evaporator or an auxiliary device of heat pump.

\section{Viability assessment of using wastewater as an energy source}

In order to assess wastewater heat recovery systems and to increase environmental awareness of the public related to the viability of their implementation in Poland, this concept was subjected to SWOT analysis. Given the difference between the qualities of raw and treated wastewater, the assessment was performed separately for wastewater carried by intercepting sewers towards a treatment plant and for medium transported towards the receiving body after the treatment process.

\subsection{Raw wastewater}

Opinions provided by a team of experts representing academic community, business enterprises, and potential users of recovered heat, were examined to determine strengths and weaknesses of heat recovery from untreated wastewater. Potential opportunities and threats were also defined, considering the specific conditions of conducting this process in Poland. SWOT analysis took into account five most important factors classified in each group. The factors are presented in Fig. 2.

One of the most important parameters determining the possibility to recover heat from wastewater is its temperature, which depends mainly on the temperature of water used. Mean annual temperature of household wastewater carried via intercepting sewers is approximately $15^{\circ} \mathrm{C}$, therefore it can be used as lower source of energy for heat pumps. Heat extracted from wastewater is most often applied for warming up water or for heating indoor spaces. In the summer it is possible to reset heat pumps into cooling operation mode, and to use untreated municipal wastewater as cold source for air-conditioning. Since energy contained in wastewater can be used in varied ways, these heat recovery systems can be applied in both small installations and in heating systems. Importantly, the use of this energy carrier makes it possible to significantly reduce dependence on availability of fossil fuels and on dominating weather conditions.

The strengths of the systems in question also include the general availability of raw wastewater, as it is generated practically in all residential or commercial buildings as well as industrial plants. Particularly favorable conditions occur in the case of trunk sewers which carry large quantities of warm wastewater. This is because flow rate is another parameter determining the possibility to utilize heat carried by wastewater.

Additional advantage of raw wastewater heat recovery systems lies in the fact that they can be located in direct proximity to heat consumers. Consequently, energy recuperated from wastewater can be utilized in the same buildings from which this medium is removed. In the most advantageous situation the installation in question is located in the vicinity of commercial facilities and shops. This is because buildings of this type are recipients of both 
heating and cooling energy [14], as a result of which energy potential of wastewater can be used most effectively.
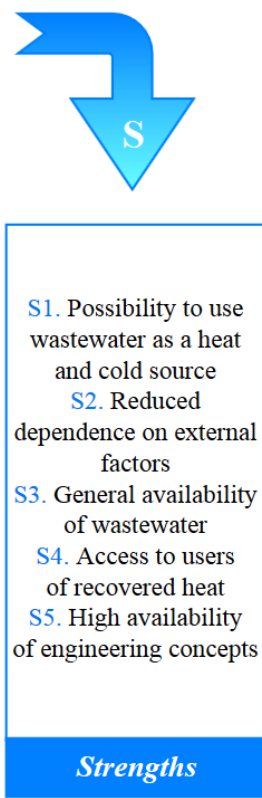
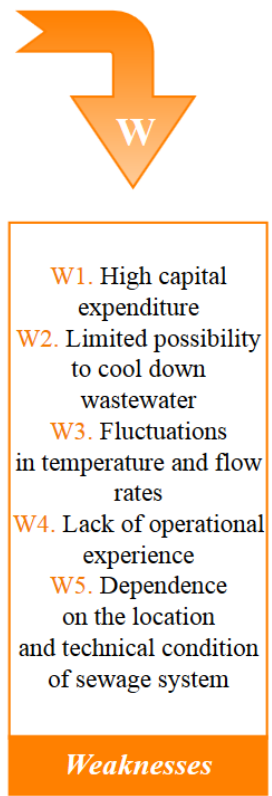

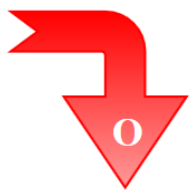
O1. Decreased use of fossil fuels and reduced emissions of pollutants
O2. Region's economic growth
O3. Increased environmental awareness
O4. Introducing relevant legal regulations and subsidies O5. Development of new engineering designs for heat exchangers
Opportunities

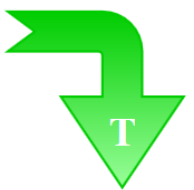

T1. Negative effects in sewage treatment process

T2. Lack of approval from potential users T3. Contamination of surfaces in heat exchangers T4. Change in residents' habits T5. Increased capacities for exploiting other energy sources

\section{Threats}

Fig. 2. SWOT analysis of exploiting raw wastewater heat recovery systems.

Another strength of such undertaking is linked with the large variety of available engineering concepts for heat exchangers, which means that they can be installed in both gravity and pressure sewers, and in specific cases also outside the sewage system. As a rule low capacity installations are applied in existing sewers. If a new sewage system is being built, or in a situation where worn out pipes are being replaced with new ones, it is possible to use heat exchangers embedded in sewage piping.

Wastewater heat recovery systems also have weaknesses. These include the significant capital expenditures linked with the cost of purchasing heat exchanger and pump, circulating pumps, and pipes of the heat exchange circuit. Notably, if a heat exchanger is situated upstream a treatment plant, the quantity of recovered thermal energy is reduced.

Weaknesses of the systems in question also include possible significant fluctuations in the temperature and flow rates of the warm medium. Such fluctuations usually occur within the diurnal and annual cycle. During a year differences in the temperature of wastewater may exceed ten degrees, which poses a technological challenge and may reduce effectiveness of an installation. In the case of systems designed for heat recovery from combined sewers there is another drawback connected with occurrence of precipitation which may significantly impact the system's effectiveness, just like in other facilities along sewage system $[15,16]$. These problems are additionally compounded by the lack of experience in operating such systems, as in Poland they have not been used previously, as well as by the fact that feasibility of heat exchanger installation is linked with the technical condition and geometry of sewers.

The opportunities linked with extracting energy from raw wastewater include the potential positive environmental effects, resulting from reduced consumption of fossil fuels and decreased emissions of combustion products.

Construction of systems based on unconventional energy sources will also contribute to increased value of real properties located in their proximity and to creating new jobs necessary for the operation of such systems. Moreover, because of the modular structure of 
heat exchangers as well as the significant length of sewers, it will be possible to expand the systems with growing demand for energy. Consequently the above factors will contribute to region's economic growth.

Rapid expansion of the systems under consideration may also be facilitated by increased environmental awareness of the public and by newly implemented legal regulations enforcing utilization of energy carried by wastewater, particularly if such laws provide for an opportunity to acquire subsidies for construction of such installations. Additional stimulus promoting recovery of energy from wastewater will come from new developments in the technology; these will include engineering designs of heat exchangers, which will be dirt resistant and far more affordable than those available today.

Factors threatening implementation of these systems include their negative effects related to sewage treatment process. A decrease in the temperature of sewage, particularly if it is long lasting, results in visibly reduced effectiveness of biological treatment to which this medium is subjected [17]. Such operations may also be challenged by a lack of approval from users of recovered heat because ignorance related to this technology and the long time needed for return on investment are discouraging factors when it comes to building sewage heat recovery systems.

Another threat for the systems discussed here is connected with the possible contamination of surfaces in heat exchangers. This is linked with the significant amount of pollutants in raw wastewater; these may deposit on the surface of heat exchangers, impairing their effectiveness. This problem is particularly significant in the case of exchangers embedded in sewers, due to the fact that it is difficult to inspect such devices.

While designing systems for recovery of heat from raw wastewater it is necessary to remember about possible changes in people's preferences related to the temperature of water used for household needs, as well as temperature in indoor spaces. Other important issues are connected with new resources of fossil fuels, such as shale gas, which are being discovered, and with the fact that devices utilizing conventional fuels are more and more effective.

\subsection{Treated wastewater}

Factors taken into account in the analysis of systems designed for heat recovery from treated effluent are listed in Figure 3. Some elements assigned to the specific groups are identical as those considered in the analysis performed for raw wastewater. Given this, the following part of the paper discusses only those factors which are characteristic exclusively for treated wastewater.

Temperature of treated effluent is slightly lower than that of wastewater flowing into facilities of treatment plant, yet it is possible to induce its decrease by even a few degrees, enabling greater recovery of energy. Moreover, fluctuations in temperature and flow rate in effluent carried towards the receiving body are insignificant, which ensures stability of the system's operation. Additionally, because the effluent contains no contaminations, it can be carried directly into heat pump evaporator, without a need to use intermediate circuits.

A drawback of the systems in question, in addition to the required significant capital expenditures and lack of experience in operating such systems, is connected with the location of treatment plants, because these are generally situated far from potential users of recovered heat. Another challenge is the limited length of discharge pipes carrying the effluent towards the receiving body, and due to this in many cases location of heat exchanger outside the treatment plant is not feasible. One more weakness of purified wastewater heat recovery systems, taken into account in the analysis, is the lack of any activities promoting operations aimed at extracting energy from wastewater. This is 
because production of electric power from biogas is far more profitable for sewage treatment plant operators than recovery of energy carried by wastewater.
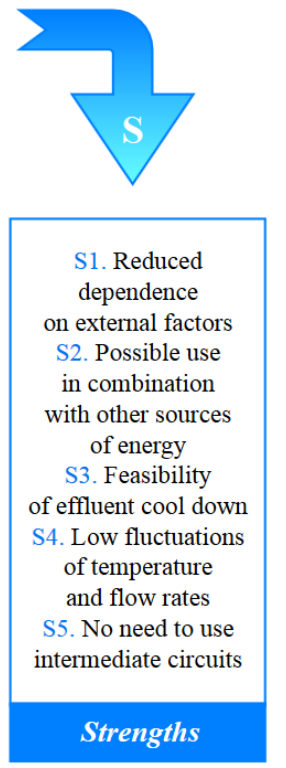
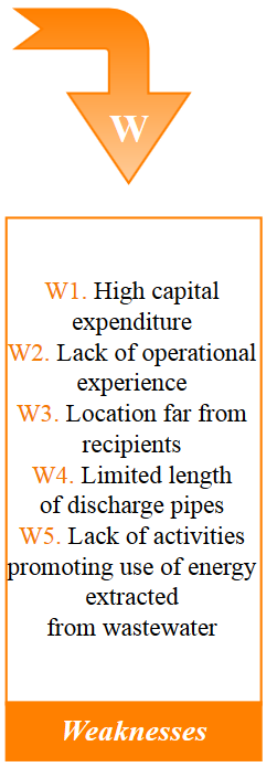

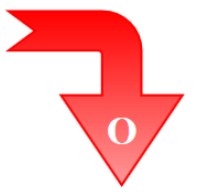

O1. Decreased use of fossil fuels and reduced emissions of pollutants O2. Increased environmental awareness

O3. Introducing relevant legal regulations and subsidies O4. Protection of the receiving body O5. Satisfying energy requirements of treatment plants

Opportunities

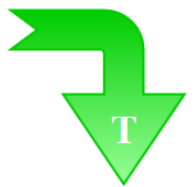

T1. Lack of approval from potential users T2. Increased capacities for exploiting other energy sources T3. Lack of capacity for expansion T4. Problems with connecting to existing energy systems in treatment plants T5. Varied sewage treatment technologies in different facilities

Threats

Fig. 3. SWOT analysis of exploiting treated wastewater heat recovery systems.

Opportunities identified as characteristic exclusively for treated wastewater include the possibility to protect the receiving body as a result of the fact that the effluent discharged has similar temperature as the water in the river. Furthermore, heat extracted from this medium may be applied for the needs of the treatment plant, for instance for warming up water used by its personnel, or for increasing the temperature of sewage flowing into the plant facilities, in order to improve effectiveness of biological treatment.

Factors recognized as threats to development of these systems include difficulties related to potential expansion due to the limited length of discharge pipes, which makes it impossible to connect additional modules of heat exchanger. Moreover, some sewage treatment plants may face problems in adjusting the designed heat recovery installation to the existing power system, particularly due to the fact that differences in sewage treatment capacity and technology in various facilities may be rather significant as a result of which standard solutions are not feasible.

\subsection{Defining development strategy for wastewater heat recovery systems}

During the next stage of the study, weights were assigned to the specific factors (separately for raw and treated wastewater), and interactions between internal and external factors were specified. Following the guidelines described in a study [10], eight supporting tables were drawn up for each type of wastewater, and based on these totals were determined for the interactions as well as products of weights and interactions. The obtained results of the analyses are shown in Tables 1 and 2. Yet, the presented findings related to both raw and treated wastewater do not provide an explicit recommendation regarding the choice of development strategy for heat recovery systems.

In the case of SWOT analysis, as well as the comprehensive SWOT/TOWS assessment of raw wastewater heat recovery systems, the highest scores were obtained for a combination of the positive factors, which is an evidence that these systems have a significant potential for development. This also shows a need for taking advantage of their 
strengths, in particular general availability of raw wastewater, in order to benefit from the potential opportunities, such as feasible improvement in the state of the natural environment. However, the results obtained for a combination of weaknesses and external factors are only slightly lower, which suggests the need for minimizing the drawbacks of these systems, most importantly the limitations related to cooling down wastewater. Furthermore, the findings presented in Table 1 show significant vulnerability of these systems to threats occurring in the future, mainly lack of approval from potential users and the possible contamination of surfaces of heat exchangers by raw wastewater. Due to this it is necessary to take action aimed at further development of wastewater heat recovery technologies and to promote sustainable management of waste energy among potential users, as this is the only approach making it possible to utilize the opportunities resulting from recovery of thermal energy from the medium transported towards treatment plant.

Table 1. The summary of analysis results for raw sewage.

\begin{tabular}{|c|c|c|c|c|c|c|}
\hline \multirow{2}{*}{$\begin{array}{c}\text { Quadrant } \\
\text { combinations }\end{array}$} & \multicolumn{2}{|c|}{ SWOT analysis } & \multicolumn{2}{c|}{ TOWS analysis } & \multicolumn{2}{c|}{ SWOT/TOWS analysis } \\
\cline { 2 - 7 } & $\begin{array}{c}\text { Sum of } \\
\text { interactions }\end{array}$ & $\begin{array}{c}\text { Sum of } \\
\text { products }\end{array}$ & $\begin{array}{c}\text { Sum of } \\
\text { interactions }\end{array}$ & $\begin{array}{c}\text { Sum of } \\
\text { products }\end{array}$ & $\begin{array}{c}\text { Sum of } \\
\text { interactions }\end{array}$ & $\begin{array}{c}\text { Sum of } \\
\text { products }\end{array}$ \\
\hline S / O & $\mathbf{3 8 / 2}$ & $\mathbf{7 . 8 0}$ & $22 / 2$ & 4.75 & $\mathbf{6 0 / 2}$ & $\mathbf{1 2 . 5 5}$ \\
\hline S / T & $16 / 2$ & 2.95 & $\mathbf{3 0 / 2}$ & $\mathbf{6 . 3 5}$ & $46 / 2$ & 9.30 \\
\hline W / O & $36 / 2$ & 7.05 & $18 / 2$ & 3.65 & $54 / 2$ & 10.70 \\
\hline W / T & $36 / 2$ & 6.55 & $24 / 2$ & 4.90 & $\mathbf{6 0 / 2}$ & 11.45 \\
\hline
\end{tabular}

Table 2. The summary of analysis results for treated sewage.

\begin{tabular}{|c|c|c|c|c|c|c|}
\hline \multirow{2}{*}{$\begin{array}{c}\text { Quadrant } \\
\text { combinations }\end{array}$} & \multicolumn{2}{|c|}{ SWOT analysis } & \multicolumn{2}{c|}{ TOWS analysis } & \multicolumn{2}{c|}{ SWOT/TOWS analysis } \\
\cline { 2 - 7 } & $\begin{array}{c}\text { Sum of } \\
\text { interactions }\end{array}$ & $\begin{array}{c}\text { Sum of } \\
\text { products }\end{array}$ & $\begin{array}{c}\text { Sum of } \\
\text { interactions }\end{array}$ & $\begin{array}{c}\text { Sum of } \\
\text { products }\end{array}$ & $\begin{array}{c}\text { Sum of } \\
\text { interactions }\end{array}$ & $\begin{array}{c}\text { Sum of } \\
\text { products }\end{array}$ \\
\hline $\mathrm{S} / \mathrm{O}$ & $24 / 2$ & 5.65 & $\mathbf{2 8 / 2}$ & $\mathbf{6 . 4 5}$ & $52 / 2$ & $\mathbf{1 2 . 1 0}$ \\
\hline $\mathrm{S} / \mathrm{T}$ & $24 / 2$ & 4.35 & $16 / 2$ & 3.90 & $40 / 2$ & 8.25 \\
\hline $\mathrm{W} / \mathrm{O}$ & $\mathbf{3 2 / 2}$ & $\mathbf{6 . 2 0}$ & $22 / 2$ & 4.50 & $\mathbf{5 4 / 2}$ & 10.70 \\
\hline $\mathrm{W} / \mathrm{T}$ & $26 / 2$ & 4.80 & $26 / 2$ & 4.95 & $52 / 2$ & 9.75 \\
\hline
\end{tabular}

A different strategy should be applied with regard to systems designed for treated wastewater heat recovery. SWOT analysis showed the largest number of interactions for the combination of weaknesses and opportunities, which suggests that viability of heat recovery process is significantly affected by the weaknesses of the systems in question. In this case significant capital expenditures and limited length of discharge pipes downstream from treatment plant are of the greatest importance, yet benefitting from potential opportunities may be hindered by the other factors classified in this group as well. On the other hand, however, the high total of products obtained for the combination of the positive factors in SWOT analysis, as well as the results of TOWS analysis for the same combination of factors, suggest that opportunities existing in the external environment, mainly the possibility to satisfy energy related needs of treatment plants, may significantly strengthen development possibilities of these systems. Ambiguity of the findings is confirmed by the results of the comprehensive SWOT/TOWS analysis, and this shows the need for further research into utilization of thermal energy carried by treated wastewater. 


\section{Conclusion}

The results of the analysis show that heat recovery systems have significant potential for development. However, in the case of raw or treated wastewater, the matter should not be approached without questioning. Utilization of heat carried by wastewater presents drawbacks, which will be difficult to overcome, given the existing legal regulations and the public's environmental awareness. Therefore, generalized recommendations with regard to utilization of energy contained in wastewater would be ill-advised or in fact impossible; consequently it is necessary to approach each project related to recovery of heat carried by wastewater on a case-by-case basis.

The conducted study has also shown that, contrary to the common opinion, raw wastewater presents more significant advantages, most notably the general availability of the resource. In Poland, however, the importance of this energy carrier is clearly underestimated, even though its utilization could increase the country's independence from external deliveries of fossil fuels and contribute to improving the state of the natural environment. Therefore it is necessary to conduct widespread promotion of this energy extraction method which will result in increased awareness related to sustainable energy systems and will contribute to gaining consumers' approval of energy extracted from wastewater. One must not forget, however, that the main purpose of sewer system is to remove and enable effective treatment of sewage, and these functions must not be neglected.

\section{References}

1. M. Starzec, J. Dziopak, M.I. Alexeev, Water and Ecology 1 (2015)

2. P. Koszelnik, Carpath. J. Earth Env. 9 (2014)

3. M. Zeleňáková, M. Čarnogurská, M. Šlezingr, D. Słyś, P. Purcz, Hydrol. Earth Syst. Sc. 17 (2013)

4. F. Hernandez-Sancho, M. Molinos-Senante, R. Sala-Garrido, Desalination 268 (2011)

5. B. Kaźmierczak, A. Kotowski, Theor. Appl. Climatol. 118 (2014)

6. K.B. Pochwat, JCEEA 63(2/II) (2016)

7. J. Danielewicz, B. Śniechowska, M.A. Sayegh, N. Fidorów, H. Jouhara, Energy 108 (2014)

8. A. Stec, S. Kordana, Resour. Conserv. Recy. 105 (2015)

9. N. Pahl, A. Richter, Swot Analysis - Idea, Methodology and a Practical Approach (GRIN Verlag, 2009)

10. K. Obłój, Strategia organizacji (PWE, Warsaw, 2007)

11. M. Ramadan, T. Lemenand, M. Khaled, Energ. Buildings 128 (2016)

12. S.S. Cipolla, M. Maglionico, Energy Procedia 45 (2014)

13. S. Kordana, D. Słyś, J. Dziopak, J. Clean. Prod. 82 (2014)

14. D. Monfet, M. Corsi, D. Choinière, E. Arkhipova, Energ Buildings 81 (2014)

15. D. Słyś, J. Dziopak, Pol. J. Environ. Stud. 20 (2011)

16. D. Słyś, A. Stec, Environ. Prot. Eng. 38 (2012)

17. O. Wanner, V. Panagiotidis, P. Clavadetscher, H. Siegrist, Water Res. 39 (2004) 